FUTURE RESEARCH DIRECTION AND CONCLUSION 


\author{
N.0. Weiss \\ Dept. of Applied Mathematics and Theoretical Physics \\ University of Cambridge \\ England
}

\title{
Abstract:
}

It is difficult to provide a comprehensive theoretical explanation for the activity of red dwarf stars. Among particular problems that are ripe for further investigation are: the production of steady, cyclic or irregular patterns of activity by nonlinear dynamo action in stars; the effect of magnetic buoyancy in producing photospheric magnetic fields; the formation of isolated flux tubes and their interaction with convection. These topics are discussed and some future lines of research are suggested.

\section{THE ROLE OF THEORY IN ASTROPHYSICS}

In astronomy, theory is generally led by observations. Even for the sun, where magnetic fields have been observed in great detail over many years, a coherent theoretical description of their structure is only beginning to emerge. The papers presented at this meeting have shown how far theoreticians are from providing an adequate explanation of magnetic activity in red dwarfs. In this review, therefore, I sha11 attempt to make some general points, and to illustrate them with specific calculations. My choice of topics is somewhat arbitrary, and slanted towards my own interests. I have tried to avoid excessive over1 ap with other papers in the se Proceedings and to focus on the interiors, rather than the atmospheres, of stars. Much of importance is therefore ignored.

It may be helpful to preface these examples with some remarks about the function of theory in astrophysics. I assume that we are dealing with situations where the basic physics is understood (thus we are not, for instance, considering the first $10^{-36}$ seconds after the big bang). The difficulty lies in applying known laws to a particular configuration. To proceed, we have to construct models that are drastically simplified in order to render them mathematically tractable. It is important to distinguish here between two different activities: producing models to 
rationalise the observations and using models to provide a basis for detailed theoretical investigations.

"Modelling" frequently implies the construction of the simplest model that fits the observational data. This is a problem in constrained optimization, shown schematically in the following flowchart.

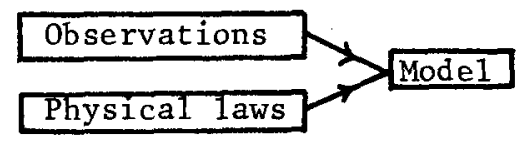

The aim is to find the simplest description that is compatible with the relevant observations, subject to the constraint that some subset of the appropriate laws should be satisfied. For example, one may constuct model atmospheres, models of the structure in magnetic loops or models of the flaring process. Such models are obviously important but I shall not discuss them further here.

A more fundamental approach is to introduce models as an aid to understanding physical processes. This leads to an iterative process, as illustrated below:

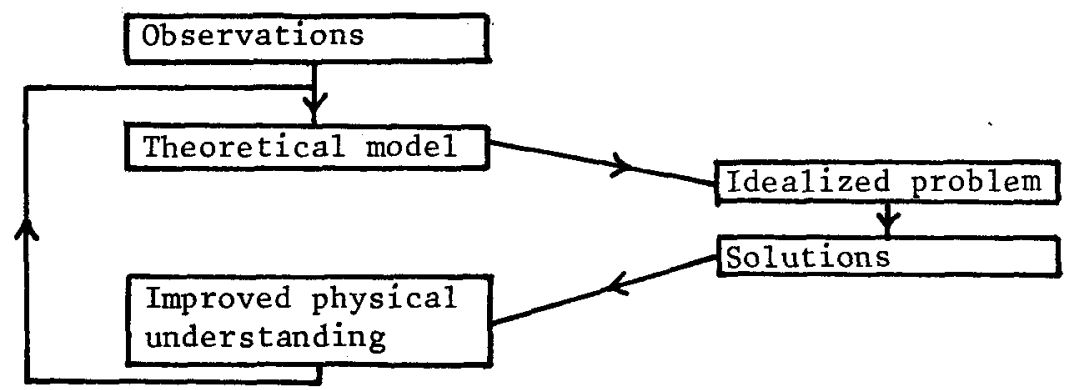

The observations are used to construct a simplified theoretical model, which provides the basis for a properly posed mathematical problem that can be rigorously solved. (whether analytically or numerically). The solutions help to improve one's physical understanding and so enable one to improve the theoretical model, thereby generating further (often more complicated) idealized problems and so forth. The success of this procedure depends critically on the interaction between theory and observation. It is vital that theoreticians should listen carefully and sympathetically to observational results but it is also important that observers should appreciate the theoretical significance of their data and organize material so that theoreticians can assimilate it. Above a11, effective communication between theoreticians and observers is needed to ensure that new and fruitful observing programs will continue to emerge. 


\section{PHENOMENOLOGICAL DESCRIPTION}

The first stage in producing a theoretical model is the development of plausible phenomenological description, which is essentially qualitative. Theoretical approaches to understanding activity in reddwarf stars have scarcely got beyond this stage. It is not obvious how to extract from the wealth of observations those features that provide the key to understanding the basic mechanisms that are involved. What is clear, however, is the need to combine results obtained from different approaches.

Studies of the sun's magnetic field have, for example, suggested that the solar cycle is driven by a dynamo located at the base of the convective zone. The most likely site seems to be the region of convective overshoot at the interface between the radiative and convective zones (e.g. Spiege1 and Weiss 1980; Schissler 1982), where a magnetic layer may be formed, as sketched in Fig. 1(a). Detailed properties of
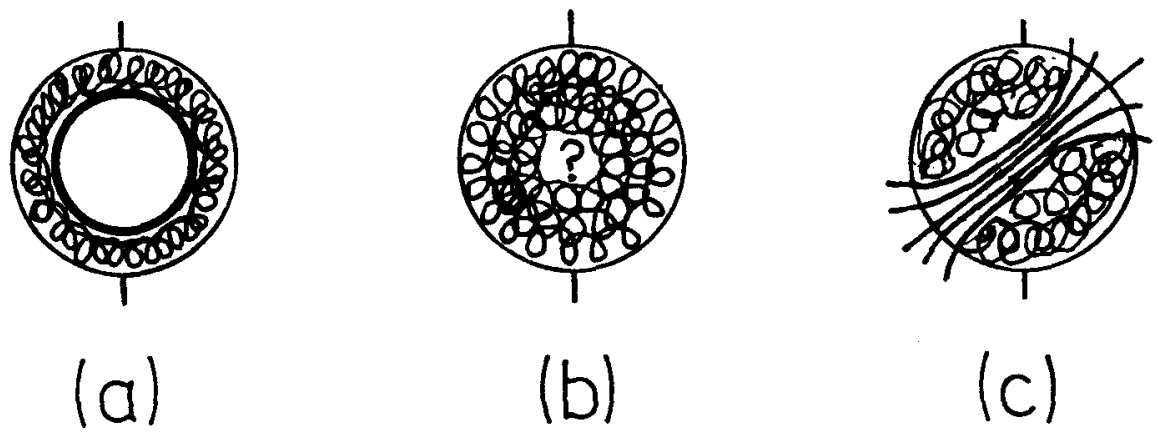

Fig. I Field configurations in magnetic stars. (a) Magnetic layer at the base of the convective zone in a G star. (b) Almost fully convective $M$ star. (c) Possible configuration for rapidly rotating RSCVn star.

such a layer have yet to be worked out. Stars of later spectral type than the sun have deeper convective zones and eventually (around M5) become fully convective as in Fig. I(b). Thus a shell dynamo model is inappropriate for a typical red-dwarf. Is there then a flux-rope dynamo that extends throughout the star, and, if so, where does the transition between the different magnetic configurations take place? Furthermore, solar activity occurs mainly in active regions, which are ephemeral and relatively small; starspots, on the other hand, are 1onglived and occupy a greater fraction of the surface. Could the fields in RS CVn variables, for instance, have the structure sketched in Fig. 
1 (c)? Such basic questions have to be answered before detailed models of magnetic stars can be investigated, and progress here depends on interpreting observational results in the light of whatever theoretical insight is available.

\section{NUMERICAL SIMULATIONS}

Although the full problem of explaining stellar magnetic fields remains intractable, insight can be gained by exploring particular aspects in some detail. Here two approaches are available: one may attempt to simulate some process or one may solve a sequence of simplified model problems. Simulations of nonlinear processes are essentially numerical and, as access to increasingly powerful computers becomes more readily available, increasingly elaborate models can be studied. To illustrate recent progress, I have chosen three examples.

Stellar dynamos. The process by which solar and stellar magnetic fields are maintained is only partially understood, as Belvedere (1982) has explained. Gilman has used the Cray computer at Boulder to study dynamo action in a simplified configuration. He models three-dimensional behaviour in a Boussinesq fluid contained between concentric spheres. The system rotates and is heated from below, so that convective motions are driven in the fluid within the region, as in the outer layers of the sun. The fluid is electrically conducting and small seed magnetic fields can be amplified and maintained (Gilman and Miller 1981). In certain parameter ranges, cyclically varying fields are produced (Gilman 1982), confirming that stellar cycles can be maintained by dynamo action, though dynamo waves progress in a poleward rather than an equatorward direction. Work on a compressible mode1 is already under way.

Turbulent magnetic fields. The laminar diffusivities used in the above calculation have values that can only be related to stellar convective zones if they are interpreted as eddy diffusivities. The behaviour of magnetic fields in a turbulent fluid, when both the ordinary Reynolds number and the magnetic Reynolds number are extremely large, has been simulated by Meneguzzi et a1. (1981), using the same Cray computer. They find that the field structure is extremely intermittent: that is to say, magnetic flux is largely confined to isolated regions, where the fields are extremely strong. Intermittent fields are to be expected in stellar convective zones (Galloway and Weiss 1981), though it is difficult to treat them explicitly in any numerical simulation of the dynamo.

Interaction of magnetic fields with granular convection. The most ambitious simulation so far attempted is Nordlund's (1981, 1982) mode1 of intergranular magnetic fields. His code incorporates the full equation of state (including ionization) and the anelastic continuity equation (so that sound waves are filtered out). The energy equation is coupled to radiative transfer in the gas and small scale turbulent dissipation is represented by additional (sub-gridscale) diffusivities. With the aid of a Cray, magnetic fields are again shown to be inter- 
mittent, with isolated flux concentrations in the downdrafts between the granules. These calculations point the way towards even more elaborate simulations in the future.

\section{SIMPLIFIED MODEL PROBLEMS}

As an alternative to large scale simulations one may pose a simplified problem that can be properly solved by some combination of analytical or numerical techniques. The main purpose of such a calculation is to investigate systematically the effect of varying certain key parameters: for instance, we would like to know how stellar activity depends on the rate of rotation of the star. Here one must be careful to distinguish between idealized models, which can have both qualitative and quantitative predictive capacity, and parametrized models, which may be illuminating but have no quantitative predictive capacity. My first example is of the latter type.

$\alpha \omega$-dynamo models. As has already been explained in these Proceedings (Belvedere 1982) one can construct an idealized description of a turbulent hydromagnetic dynamo in which the magnetic field has a poloidal component described by the vector potential $\mathrm{A}$, and a toroidal component $B$. The poloidal field is regenerated by the $\alpha$-effect, depending upon the helicity of the flow (Moffatt 1978, Parker 1979), while the toroidal field is maintained by differential rotation. The growth of the field is governed by the equations

$$
\frac{\partial A}{\partial t}=\alpha B+n D^{2} A, \quad \frac{\partial B}{\partial t}=r \underset{\sim}{B} \cdot \nabla \Omega+D^{2} B
$$

and the crucial parameter is the dimensionless dynamo number

$$
\Phi=\frac{\alpha v^{\prime} L^{3}}{n^{2}} \text {. }
$$

Here $\Omega$ is the angular velocity and $v^{\prime}$ a typical value of the velocity gradient, $\eta$ is a turbulent diffusivity, $L$ a characteristic length and $D^{2}$ the Stokes diffusion operator. Linear theory tells us that the field grows exponentially if $\mathscr{D}$ is sufficiently large. What behaviour can be expected in the nonlinear domain?

The last few years have seen a rapid advance in understanding different patterns of nonlinear behaviour. To explore the behaviour of nonlinear dynamos it is convenient to take a simple parametrized model. Consider one-dimensional dynamo waves, travelling along the $x$-axis of Cartesian coordinates, with a magnetic field $\underset{\sim}{\mathrm{B}}=(0, \mathrm{~B}, \partial \mathrm{A} / \partial \mathrm{x})$ and $\mathrm{a}$ velocity $\mathrm{V}=(0, \mathrm{v}(\mathrm{z}), 0)$ (Parker 1979). Then nonlinear waves can be modelled by a system of three complex ordinary differential equations,

$$
\begin{aligned}
& \dot{\mathrm{A}}=2 \mathrm{~dB}-\mathrm{A}, \\
& \dot{\mathrm{B}}=\mathbf{i} A-\frac{1}{2} \mathrm{iA} *_{\omega}-\mathrm{B},
\end{aligned}
$$


(Cattaneo et al. 1982), where $\omega$ represents the nonlinear effect of the Lorentz force on the velocity shear and $V$ is a constant. The system (3) has a static solution $A=B=\omega=0$, which becomes unstable to oscillatory solutions (dynamo waves) when the parameter $D=1$. For $\mathscr{D}>1$ there are periodic solutions which are themselves unstable for $\nu<1$. When $\nu=0.5$ there are successive bifurcations to doubly and to triply periodic solutions, followed by a period-doubling cascade and chaotic (aperiodic) behaviour. Fig. 2 shows an example of an aperiodic solution, for $D=16$; the recurrent episodes of reduced activity correspond to Maunder minima. Successive bifurcations, leading to chaos, are a feature of many nonlinear systems and similar behaviour must be expected in more elaborate models of stellar dynamos.

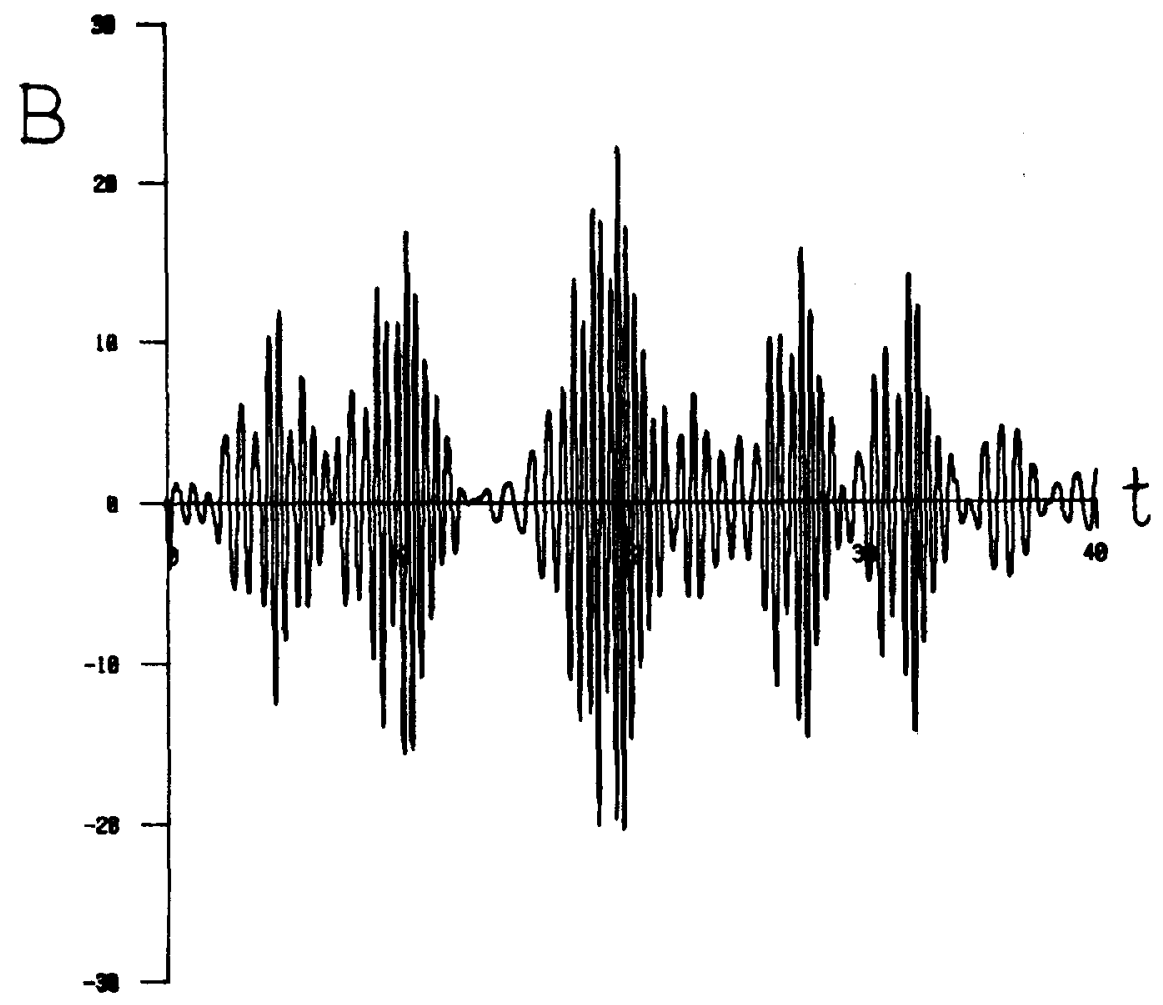

Fig. 2 Chaos in solutions of the model dynamo equations. The toroidal magnetic field $B$ as a function of time for $v=0.5, D=16$, showing aperiodic oscillations with episodes of reduced activity (Cattaneo et al. 1982). 
Formation of flux ropes by magnetic buoyancy. In the sun, magnetic flux tubes emerge through the photosphere to form active regions. These flux tubes could be formed if the magnetic layer in Fig. 1(a) becomes unstable, as shown schematically in Fig. 3 (Parker 1979). The instabi1ities can be studied for an idealized model: suppose that there is a

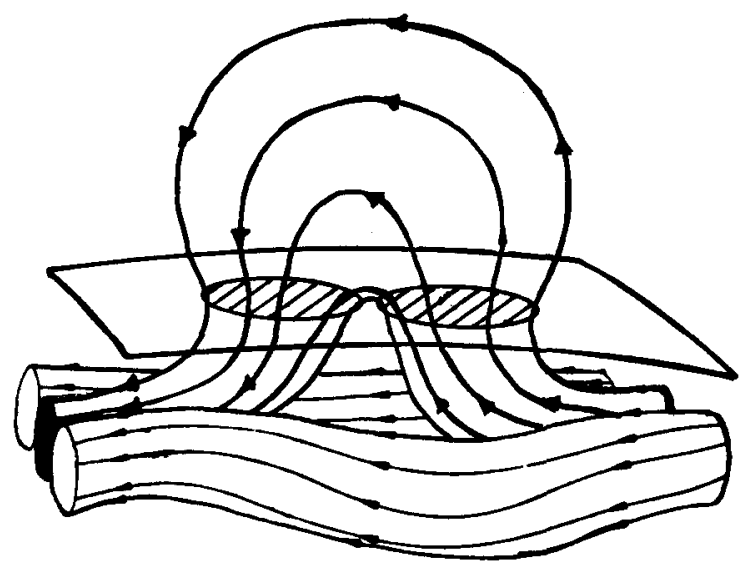

Fig. 3 Sketch showing the formation of a flux rope as a result of instabilities driven by magnetic buoyancy in a magnetic layer (after Parker 1979).

field $\mathrm{B}_{0}=\left(0, B_{0}(z), 0\right)$ referred to Cartesian coordinates with the $z$-axis pointing upwards. Such a field is unstable to modes varying as $\exp (i k \cdot r)$, with $k_{x} \geq k_{z} \gg k_{y}$, if $d\left(B_{o}{ }^{2}\right) / d z<0$. More generally, there are complicated doubly-diffusive instabilities, depending on the relative rates at which magnetic fields, heat and momentum diffuse through the medium (Acheson 1978, 1979; Schmitt and Rosner 1982; Hughes 1982), which can be studied within the Boussinesq approximation (Spiegel and Weiss 1982; Hughes 1982). Typically the most unstable modes have a much longer wavelength in the direction parallel to $\mathrm{B}_{\mathrm{O}}$ than in the transverse direction and they might therefore be expected to develop into the configuration sketched in Fig. 3. Thus magnetic buoyancy is an essential ingredient of any theory that relates surface activity to deep-seated dynamos. Work on the nonlinear problem has, however, only just begun (R. Rosner, private communication).

Isolated flux tubes. In the solar photosphere magnetic fields are extremely intermittent, as might be expected from the simulations that were described above. Intense magnetic fields are confined to slender isolated flux tubes, which are partially evacuated so that the magnetic pressure within is contained by the pressure of the external gas (Parker 1979). Thin tube models have been investigated by Spruit (1981), who identified an instability that leads to collapse and partial evacuation; 
and the structure of the field within the tubes has been described by Deinzer et al. (1982). The se models adopt a parametrized representation of convection in the ambient gas. A proper treatment would combine these effects (important when the magnetic field exerts a pressure comparable to that of the ambient gas) with a better description of convection in the surrounding region, as in Nordlund's simulation.

The kinematic interaction of weak magnetic fields with convection has been investigated in some detail. In two dimensions, or with axial symmetry, flux is expelled from the convection cells and concentrated into isolated sheets or tubes (Galloway and Weiss 1981). In three dimensions the story is, however, much more complicated (Galloway and Proctor 1982; Arter et a1. 1982). When there is an imposed horizontal field, Arter (1982) has shown that closed magnetic loops are formed. As a result, the horizontally averaged field is reversed in the upper part of a convecting layer, as shown in Fig. 4. Moreover, flux associated with the reversed field may be greater than that originally imposed. This result upsets many preconceptions about the structure of magnetic fields in stellar convection zones.

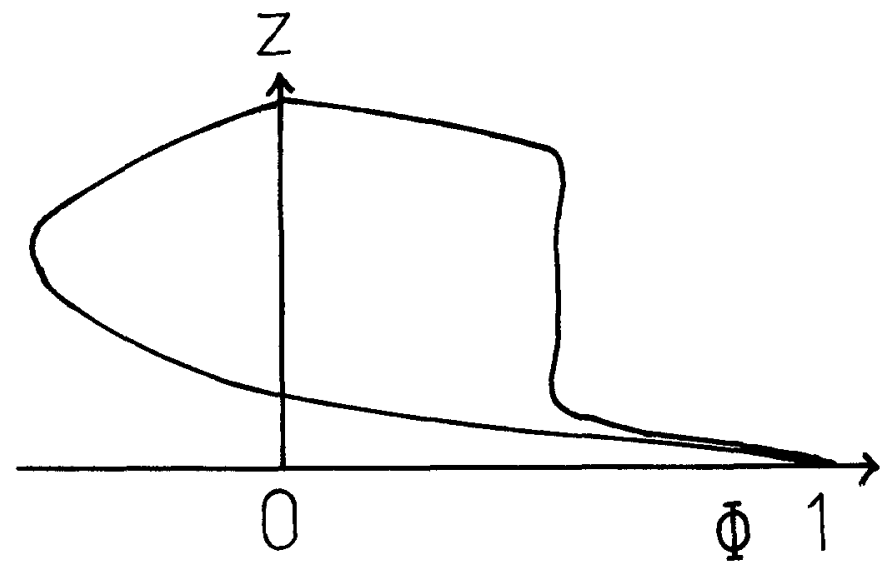

Fig. 4 The horizontally averaged magnetic flux $\Phi$ as a function of height, for three-dimensional convection (left-hand curve) and two-dimensional convection (right-hand curve). Note the field free region which is present for the two-dimensional case, and the reversed field in the three-dimensional case (Arter 1982). 
Flux concentration produces strong magnetic fields and the Lorentz force becomes important in the equation of motion. Dynamical calculations for convection with two-dimensional or axial symmetry show that isolated tubes and sheets of flux persist (Proctor and Weiss 1982), and some preliminary results are now available for three-dimensional magnetoconvection in a Boussinesq fluid (W. Arter, private communication). Cattaneo (1982) has investigated compressible magnetoconvection and confirmed that flux sheets are partially evacuated in a simple twodimensional model. A systematic study of three-dimensional compressible magnetoconvection is, however, yet to come. After that, it may be possible to combine treatments of convection in an imposed magnetic field with a description of the dynamo that generates the basic field. At present, owing to the intermittent structure of the field, any such computation seems beyond the bounds of feasibility.

\section{FUTURE PROSPECTS}

The examples that I have described illustrate the different approaches taken by theoreticians, as well as the difficulties that they face. Viewing the problem as a whole, one can also glimpse the way ahead. The one certain feature is that we shall see much more of the same, or similar, investigations. I believe that the emphasis will move from linear to nonlinear behaviour, and so from analysis to computation. Fortunately, advances in bifurcation theory and the theory of dynamical systems are making it easier to interpret the results of such numerical experiments.

As the subject progresses, connections will be established between the different types of theoretical model that I have described. More physics 11 be fed into the idealized models; for instance, I expect to see fully compressible, three-dimensional computations, utilizing experience gained from plasmas in fusion research. Eventually, the idealized models will merge with simulations, so that we can understand the effect of varying parameters as well as the detailed behaviour of a realistic system. Finally, feedback to phenomenological descriptions may allow us to clarify what happens in a star. Then a coherent dynamo will emerge, incorporating intermittent fields; the role played by rotation will be understood and the origin of the sun's magnetic field, at any rate, wil1 be explained.

Beyond that, we need a global description of the evolution of main sequence magnetic stars. We know that the surface rotation rate is correlated with activity and that angular momentum is lost owing to magnetic braking. The internal rotation rate is much less certain, though nonuniform rotation is to be expected. Indeed, solar oscillations suggest that the core of the sun rotates from 2 to 10 times faster than the surface (Claverie et a1. 1981; Gough 1982). So how is the all-important surface rotation rate related to the angular velocity within the star? In the radiative zone, the gradient of $\Omega$ is limited by instabilities driven by differential rotation. The linear theory of these instabilities 
has been studied in some detail (e.g. Knobloch and Spruit 1982) but their nonlinear development is unclear. Thus we do not know how the angular velocity evolves. Nor do we understand how magnetic behaviour changes with spectral type. What is the effect of deep convective zones on the distribution of angular momentum? Are $M$ dwarfs more or less uniformly rotating? These questions have to be answered in the future.

\section{REFERENCES}

Acheson, D.J. : 1978, Phi1. Trans. Roy. Soc. A 289, pp.459-500. Acheson, D.J. : 1979, Solar Phys. 62, pp.23-50.

Arter, W. : 1983, in "Solar and Stellar Magnetic Fields" (IAU Symp. No.102), ed. J.0. Stenflo, Reide1, Dordrecht.

Arter, W., Galloway, D.J. and Proctor, M.R.E. : 1982, Mon. Not. Roy. Astr. Soc., in press.

Belvedere, G. : 1983, in these Proceedings.

Cattaneo, F. : 1982, in preparation.

Cattaneo, F., Jones, C.A. and Weiss, N.O. : 1983, in "Solar and Stellar Magnetic Fields" (IAU Symp. No.102), ed. J.o. Stenflo, Reide1, Dordrecht.

Claverie, A., Isaak, G.R., Mcleod, C.P. and van der Raay, H.B. : 1981, Nature 293, pp.443-445.

Deinzer, W., Hensler, G., Schmitt, D., Schlssler, M. and Weisshaar, E. : 1983, in "Solar and Stellar Magnetic Fields" (IAU Symp. No. 102), ed. J.0. Stenf1o, Reide1, Dordrecht.

Galloway, D.J. and Proctor, M.R.E. : 1982, in "Planetary and Stellar Magnetism", ed. A.M. Soward, Gordon and Breach, London.

Galloway, D.J. and Weiss, N.0.: 1981, Astrophys. J. 243, pp.945-953.

Gilman, P.A. : 1983, in "Solar and Stellar Magnetic Fields" (IAU

Symp. No. 102), ed. J.0. Stenflo, Reide1, Dordrecht.

Gilman, P.A. and Miller, J. : 1981, Astrophys. J. Supp. 46, pp.211-238. Gough, D.0. : 1982, Nature 298, pp.334-339.

Hughes, D.W. : 1982, in preparation.

Knobloch, E. and Spruit, H.C. : 1982, Astron. Astrophys., in press.

Meneguzzi, M., Frisch, U. and Pouquet, A. : 1981, Phys. Rev. Lett. 47, pp. 1060-1064.

Moffatt, H.K. : 1978, "Magnetic Field Generation in Electrica1ly Conducting Fluids", Cambridge University Press.

Nordlund, A. : 1981, Astron. Astrophys. 107, pp.1-10.

Nordlund, A. : 1983, in "Solar and Stellar Magnetic Fields" (IAU Symp. No. 102), ed. J.0. Stenflo, Reide1, Dordrecht.

Parker, E.N. : 1979, "Cosmical Magnetic Fields", Clarendon Press, Oxford.

Proctor, M.R.E. and Weiss, N.0. : 1982, Rep. Prog. Phys., in press.

Schmitt, J. and Rosner, R. : 1982, Astrophys. J., in press.

Schlissler, M. : 1983, in "Solar and Stellar Magnetic Fields" (IAU

Symp. No. 102), ed. J.0. Stenf1o, Reide1, Dordrecht.

Spiege1, E.A. and Weiss, N.0. : 1980, Nature 287, pp.616-617.

Spiege1, E.A. and Weiss, N.0.: 1982, Geophys. Astrophys. Fluid Dyn., in press. 
Spruit, H.C. : 1981, in "The Sun as a Star", ed. S.D. Jordan, pp.385-412, NASA SP-450, Washington.

\section{DISCUSSION}

van Leeuwen: Do you consider it possible that by means of magnetic braking a $\mathrm{K}$ star could lose $99 \%$ of its angular momentum in 10-20 million years as we observe for the Pleiades?

Weiss: I do not know of anyone having and adeauate model of magnetic braking or understanding how it could be as efficient as that. Clearly, however, the picture that we have implies a rather gradual loss of angular momentum through magnetic braking. The timescale you mention is not inconceivable but it would require a very vigorous form of magnetic activity so that the lever arm for magnetic braking would be much greater.

Rosner: I would like to comment that it is probably difficult to know something about the angular momentum of a star. All you have is a measure of the spindown of the surface. So one does not know anything about the angular momentum of the whole star unless one assumes something about differential rotation as a function of depth. So it is very model independent.

Weiss: It is possible that the magnetic field would be confined to a skin near the surface which is, as a result, rotating very slowly, while the core is whizzing round. However one is reluctant to assume this unless it is forced on one by observations.

Lang: One of your first diagrams showed observations leading to models and so on. I wonder if you should not complete that feedback loop. For instance, does the production of flux ropes by magnetic buoyancy reproduce any observable properties of sunspots? For instance, is an isolated flux tube observable? How thin would it be? What is its magnetic field strength?

Weiss: I think that at oresent the argument goes only the one way. We have gone from the observations to a phenomenological description. We observe flux tubes emerging as active regions at the surface of the Sun which suggests that some process of this type is occurring. For a variety of reasons some of us think that they originate very down in the convective zone. The only mechanism that one can imagine for driving this process is an instability driven by magnetic buoyancy. Although it was understood that convection could produce locally magnetic fields through the flux expulsion mechanism, nobody has supposed that intense magnetic fields, virtually completely evacuated, could exist at the surface of the Sun 
until such fields were discovered. Intense magnetic fields were discovered 10 years ago and they have been intensively investigated. We know there are isolated flux tubes with fields of $1500 \mathrm{G}$ or so and magnetic pressure is comparable with external gas pressure at the Sun's surface. This has led Spruit and others to seek mechanism for an instability... (part of recording lost)... But $I$ think it is true to say that in these cases observations led theory.

Catalano: You nosed the question how does an M star rotate? This arises because they may be fully convective and so on. We have evidence that the rotation period of a main sequence star changes with some power of the mass and indirect evidence, from $\mathrm{Ca} \mathrm{K}$-line behaviour (Catalano and Marilli, this volume) in the Hyades and Pleiades on the decay of rotation with time, that the rotation speed changes exponentially with time and with a powerlaw denendence on mass. So perhaps this could be an answer to the question you pose?

Weiss: This is very helpful. What one would expect from simple considerations is that magnetic braking might be less effective for stars with deeper convective zones. Since one could not decelerate an outer shel1. Rather one would have to decelerate to whole star.

Gibson: Would not tidal forces in the RS. CVn stars have eliminated differential rotation, at least while they were on the main sequence?

Weiss: Indeed yes. I would have thought that in close binaries one would have thought that tidal forces would have forced at least rough co-rotation, although there might be a modest degree of differential rotation within the convective zone.

Gibson: So as much an object evolves off the main sequence it is possible that the inner parts would rotate more rapidly again than the surface. Would this rapidly rotating repion incornorate some of the convective zone or would it be in the radiative zone?

Weiss: That is a question which is beyond my capacity to answer. One should need to know more about stellar structure than I do.

Rodonò: I would say that certainly there would be competition between differential rotation and gravitational effects in close binaries. There are two papers by Scharlemann in Astrophysical Journal (1981 and 1982) which treat the effect of tidal coupling on the differential rotation of RS CVn stars. 